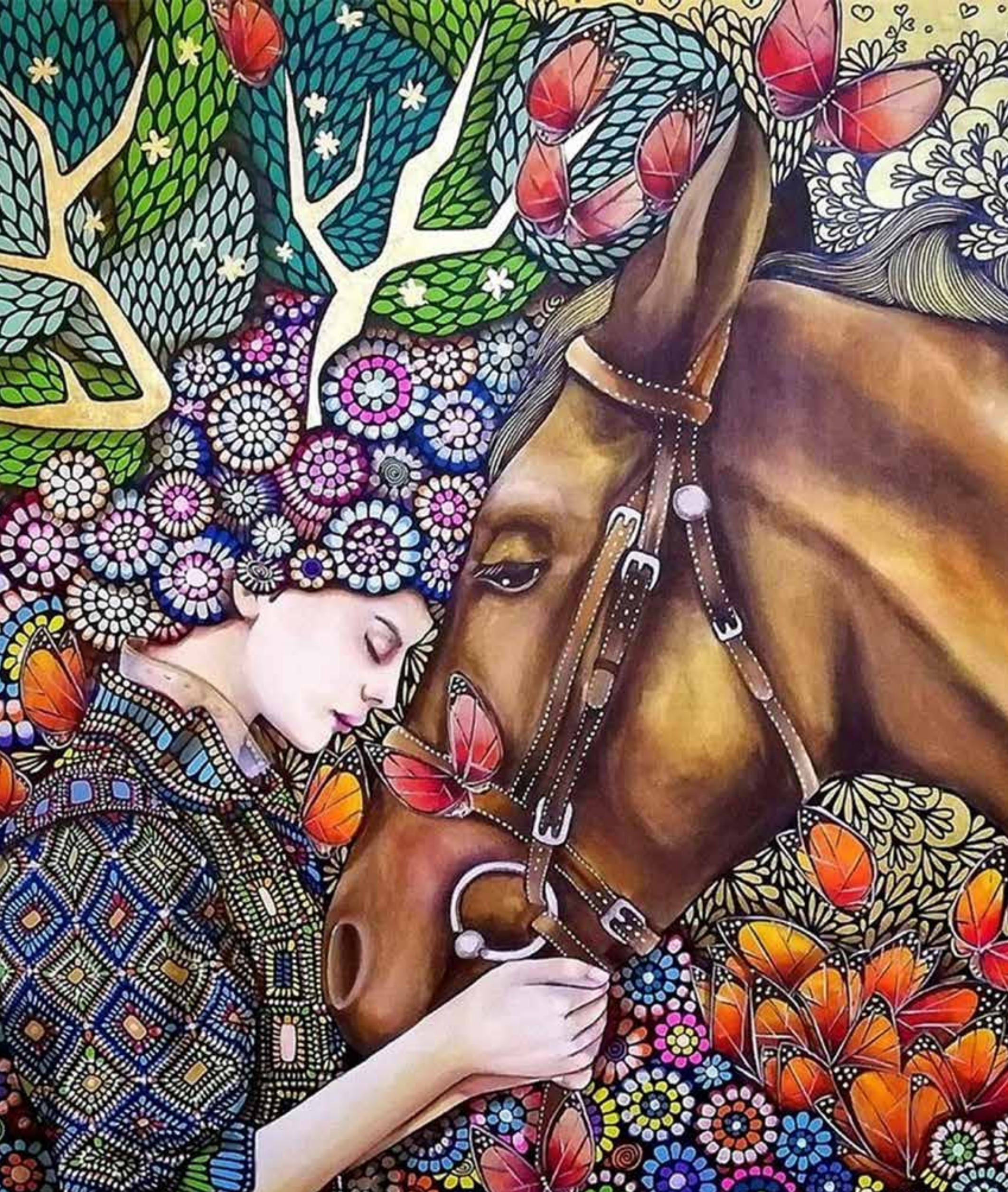




\section{EL MIGRANTE DE TIERRA LEJANA}

El migrante de tierra lejana camina andante y perseverante hacía una tierra distante y añorada.

Tiene la esperanza de algún día llegar sano a la tierra prometida que cambiara su destino, un migrante lejano.

Cada migrante sufre la nostalgia de dejar a su familia, no importa cuántas lágrimas, penas y sufrimiento pase en su camino el migrante seguirá adelante con la frente en alto sin perder el horizonte de su destino.

El norte es su esperanza para cambiar su vida, Jesucristo ilumina su camino el migrante lejano llegará a su destino. 


\title{
2. Nuevos patrones de migración centroamericana a Estados Unidos: La caravana en Piedras Negras, Coahuila, México, 2019
}

\author{
Felipe Javier Uribe-Salas ${ }^{1}$ \\ Juan Parra Ávila ${ }^{2}$ \\ Juan Felipe Mayo Carrillo 3
}

DOI: https://doi.org/10.5377/pdac.v16i0.10225 Recibido: 20/05/2019 - Aceptado: 9/10/2019

Resumen: El objetivo de la investigación fue caracterizar socio-demográficamente a integrantes de la caravana de migrantes centroamericanos en Piedras Negras, Coahuila. Metodológicamente se aplicó un cuestionario a una muestra de 540 personas, con la información se realizó un análisis descriptivo e inferencial cuyas variables fueron estratificadas por sexo. Se encontró una población joven, con 2.5 hombres por cada mujer, originarios principalmente de Honduras, con escolaridad dominante de primaria, principalmente solteros, con ocupaciones que en su mayoría no requieren formación escolar. Las mujeres tienden, en mayor proporción que los hombres, a viajar acompañadas, con dos o más personas, con un mayor número de menores, con un mayor número de familiares, con su pareja y con hijos. La principal razón de salir de su país fue la falta de trabajo, la mayoría viaja a Estados Unidos (EU) por primera vez, tenían familiares en EU en más de la mitad de los casos, más de dos tercios buscaba solicitar asilo en EU y la gran mayoría no tenía el deseo de regresar a su país de origen.

En conclusión, asistimos a una nueva estrategia de la migración centroamericana hacia EU a través de México que, en términos socio-demográficos, apoya la hipótesis de una teoría expulsora por razones económicas y de seguridad. Se plantean nuevas estrategias con enfoque de género para realizar un viaje más seguro y muestra que existe un objetivo claro de viajar hacia EU con pocos casos para residir en México o para regresar al país de origen.

Palabras clave: caravana migrante, Centroamérica, dinámica migratoria, Piedras Negras.

\section{New patterns of Central American migration to the United States: The caravan in Piedras Negras, Coahuila, Mexico, 2019}

Abstract: The objective of this study was to perform a socio-demographic characterization of Central American migrants' caravan in Piedras Negras, Coahuila. In methodological terms a questionnaire was applied to a sample of 540 persons in order to perform descriptive and inferential analysis of variables that were stratified by sex. A young population was found with 2.5 men for each woman. People were mainly: from Honduras, with primary schooling, single, with occupation that mostly does not require school training. Women tend in a greater proportion than men to travel accompanied, with two or more people, with a greater number of children, with a greater number of relatives, with their partners and with children. The main reason for leaving their country was the lack of jobs. Most people travel to the United States (US) for the first time and have relatives in the US in more than a half of cases. More than two thirds sought asylum in the US and the vast majority did not have the desire of returning to their country of origin.

In conclusion, a new strategy of Central America migration to the US through Mexico has been described. In socio-demographic terms, the results support the hypothesis of an expulsion's theory for economic and security reasons. New strategies are proposed to make a safe trip along Mexico through a gender approach. The results also highlight a clear objective of traveling from Central America to the US with few cases to reside in Mexico or return to their country of origin.

Keywords: migrant caravan, Central America, migration dynamics, Piedras Negras.

\section{Introducción}

En los últimos años la migración centroamericana hacia Estados Unidos (EU), a través de México, ha tomado notoriedad en los medios de comunicación y es un tema de discusión en la academia. Entre algunos indicadores considerados destaca la presen- cia de un incremento significativo en la migración a EU de menores no acompañados provenientes en su mayoría de Centroamérica (Pierce, 2015, p. 1). Asimismo, se ha reportado un crecimiento de la población inmigrante centroamericana en EU pues entre el año 2000 y 2015 se presenta un incremen-

1 Dr. en Ciencias Sociales. El Colegio de la Frontera Norte, Piedras Negras, Coahuila, México, e-mail: fjuribe@colef.mx 2 M.C. en Planeación Agropecuaria, Especialidad en Estudios México-Estados Unidos, El Colegio de la Frontera Norte, e-mail: parra03@hotmail.com

3 Ing. En Sistemas Computacionales, Especialidad en Estudios México-Estados Unidos, El Colegio de la Frontera Norte, e-mail: felipe2988@colef.mx 
to de $40 \%$ (Lesser y Batalova, 2017, p. 2). De igual manera se registra un incremento de la migración centroamericana en tránsito por México hacia EU estimada a través de los montos de eventos de repatriación desde México que pasaron de 61,000 en 2009 a 132,000 en 2015 (González, Zapata y Anguiano, 2017, p. 223).

Diversos autores analizan la implementación de políticas migratorias restrictivas tanto en México como en EU (Schiavon, 2015; Castillo y Toussaint, 2015), que han influido en la reconfiguración de las rutas de desplazamiento de los migrantes centroamericanos a través de México (Martínez, Cobo y Narváez, 2015 , p. 152). En ese sentido, los migrantes centroamericanos han enfrentado riesgos y peligros a lo largo de las rutas que los conducen al norte de México que en muchas ocasiones se imbrican con las rutas del narcotráfico. Este último fenómeno se asocia a una concomitante incursión de organizaciones criminales que se dedican en paralelo al tráfico de personas (París, Ley y Peña, 2016, p. 23).

Estas características de la migración centroamericana hacia EU se presentan como un fenómeno complejo dominado por factores económicos, políticos, sociales y ambientales. Según López Recinos (2013) Honduras presenta características propias que han contribuido a la configuración de una historia política turbulenta. Sobresalen una dependencia económica y subordinación política a EU, una enorme acumulación de la riqueza y poder político en pequeñas élites y una inseguridad y vulnerabilidad social que en conjunto han impedido un desarrollo sostenible con sólidas bases democráticas (p. 67).

La historia de la migración reciente de El Salvador está ligada en gran medida a la guerra civil que se desarrolló en ese país entre 1980 y 1992, a la aplicación de reformas neoliberales que disminuyeron el gasto público y empobreció a la población, una subordinación política y militar a EU, la deportación de jóvenes salvadoreños que pertenecían en ese país a pandillas y que al regresar a El Salvador aceleraron las tendencias de la violencia, así como la presencia de desastres naturales, fenómenos que en su conjunto alimentan los flujos de persona que abandonan el país (Ramos et al., 2013; Zúñiga Núñez, 2016; Menjívar y Gómez, 2018).

En el caso de la migración guatemalteca por México existen antecedentes que se remontan a períodos anteriores a la conformación de la frontera entre am- bos países. Sin entrar en detalles se destacan los movimientos temporales de trabajadores agrícolas guatemaltecos, el conflicto armado entre la guerriIla, el ejército guatemalteco y grupos paramilitares entre 1960 y 1996 que generó el desplazamiento de miles de guatemaltecos a México, EU y Canadá, la reactivación económica tras los acuerdos de paz no fue suficiente para recuperar la deteriorada economía ni capaz de reconstruir los tejidos sociales de tal manera que sectores importantes de la población empezaron a buscar solución a sus problemas fuera de las fronteras de su país (Castillo y Toussaint, 2015, p. 72).

Es en este contexto sociohistórico en que asistimos a un nuevo fenómeno demográfico representado por la conformación de contingentes de migrantes de origen centroamericano que se desplazan en caravana, fenómeno que ya ha sido documentado con antelación en México (Martínez Hernández-Mejía, 2018; (El Colef, 2018). El presente trabajo tiene por objeto realizar una caracterización socio-demográfica de las personas entrevistadas en el marco de la caravana que arribó en febrero de 2019 a la ciudad de Piedras Negras (PN), Coahuila, desde Centroamérica.

\section{Metodología}

\subsection{Lugar del estudio}

La caravana de migrantes centroamericanos arribó a Piedras Negras ${ }^{4}$ (PN) el lunes 4 de febrero por la tarde a bordo de 51 autobuses que partieron por la mañana de la capital del estado en Coahuila. Estos migrantes que sumaron aproximadamente 1,800 personas fueron alojados en dos naves industriales de la antigua empresa Maquiladora de Componentes y Ensambles S. A. (Macesa) previamente habilitadas como albergue por el ayuntamiento de la ciudad.

\subsection{Diseño del estudio}

Mediante un estudio transversal se aplicó el cuestionario titulado "Condiciones de movilidad y estancia de la población de la caravana migrante" diseñado previamente para su aplicación en la caravana que llegó a la ciudad de Tijuana, Baja California, en noviembre de 2018 (El Colef, 2018). El cuestionario contenía 21 preguntas que indagaba sobre las siguientes características socio-demográficas de los migrantes: edad, sexo, país de origen,

4 La ciudad está localizada en la frontera norte del estado de Coahuila, México, el cual colinda con el sur del estado de Texas, EU. 
lugar de residencia al iniciar el viaje, escolaridad, última ocupación ${ }^{5}$, estado marital, si viajaba solo o acompañado, si llegó en la caravana a PN, lugar en que se sumó a la caravana, razones por las que salió de su país, si es la primera vez que viaja a Estados Unidos, las veces que ha intentado cruzar a Estados Unidos, las veces que ha logrado cruzar a ese país, si tiene familiares en Estados Unidos, con cuántas personas viaja, si viaja con familiares, si viaja con hijos, le gustaría regresar a su lugar de origen, razones por las que no le gustaría regresar, si ha iniciado trámites migratorios en México, cuál trámite, si ha solicitado trabajo en PN, si tiene problemas de salud que ameritan atención, en caso de ser mujer si estaba embarazada (Consultar Anexo $\left.N^{\circ} 1\right)$.

\subsection{Tamaño de la muestra y selección de la po- blación}

Se calculó un tamaño de la muestra de 384 cuestionarios tomando en cuenta un nivel de confianza de $95 \%$, una precisión absoluta específica de $5 \%$ y una prevalencia del factor de interés de $50 \%$ (Lwanga y Lemeshow \& WHO, 1991), sin embargo, se aplicaron 540 cuestionarios. La aplicación del cuestionario se realizó los días 11 y 12 de febrero. El abordaje de los migrantes se hizo de la participación de un equipo de 10 encuestadores divididos en dos para trabajar uno en cada nave. Se ingresó a la nave correspondiente y se repartieron los encuestadores en círculo para iniciar la aplicación de las encuestas. En forma aleatoria se invitó a un migrante a contestar el cuestionario y al terminar se siguió seleccionando candidatos en el espacio contiguo. Se seleccionaron personas de 18 años de edad en adelante. La tasa de no respuesta fue de diez por ciento. El segundo día se aplicaron 203 para un total de 535 cuestionarios terminados. A ese total se agregaron cinco cuestionarios de la prueba piloto.

\subsection{Plan de análisis}

Con la información obtenida en el cuestionario se construyeron mapas para representar los departamentos de residencia de los migrantes en Centroamérica al momento en que se unieron a la caravana y también para representar los departamentos de los países de Centroamérica y los estados en México donde los migrantes se unieron a la caravana.
Se realizaron estadísticas descriptivas de las variables socio-demográficas y se realizaron análisis de asociación entre el sexo de los entrevistados y las variables socio-demográficas utilizando la prueba de Chi cuadrada.

\section{Discusión de resultados}

\subsection{Características sociodemográficas de la po- blación migrante}

Del total de personas entrevistadas 150 fueron mujeres $(27.8 \%)$ y 389 hombres $(72.2 \%)$, mostrando una razón de 2.5 hombres por cada mujer. El promedio de edad de mujeres y hombres fue muy similar de 29.9 y 29.5 años, respectivamente. El Gráfico $N^{\circ} 1$ muestra la distribución de la edad estratificada por sexo, donde se aprecia una alta proporción tanto de mujeres como de hombres en el grupo de 20 a 24 años seguido por el de 25 a 29 años que acumulan en conjunto la mitad de las observaciones.

Gráfico N$^{\circ} 1$ Pirámide poblacional de los integrantes de la caravana de migrantes centroamericanos en febrero (valores absolutos), 2019

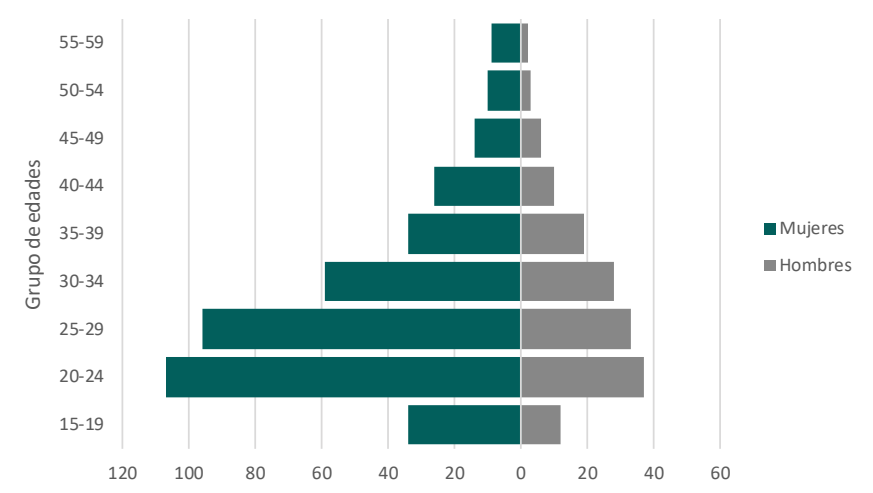

Fuente: elaborada con resultados del cuestionario aplicado a migrantes en Piedras Negras, Coahuila, en febrero de 2019.

Más de dos tercios provenían de Honduras (78.5\%), seguidos con un poco más de un décimo de El Salvador $(12.7 \%)$. Frecuencias menores se presentaron en migrantes de Guatemala (6\%) y otros países $(2.8 \%)$. No se presentaron diferencias por sexo según país de origen.

5 Para la clasificación de ocupaciones se utilizó el Sistema Nacional de Clasificación de Ocupaciones 2011 (SINCO). México, INEGI, 2011. 
Mapa $N^{\circ} 1$ Departamentos de residencia en Centroamérica de los migrantes al momento en que se unieron a la caravana

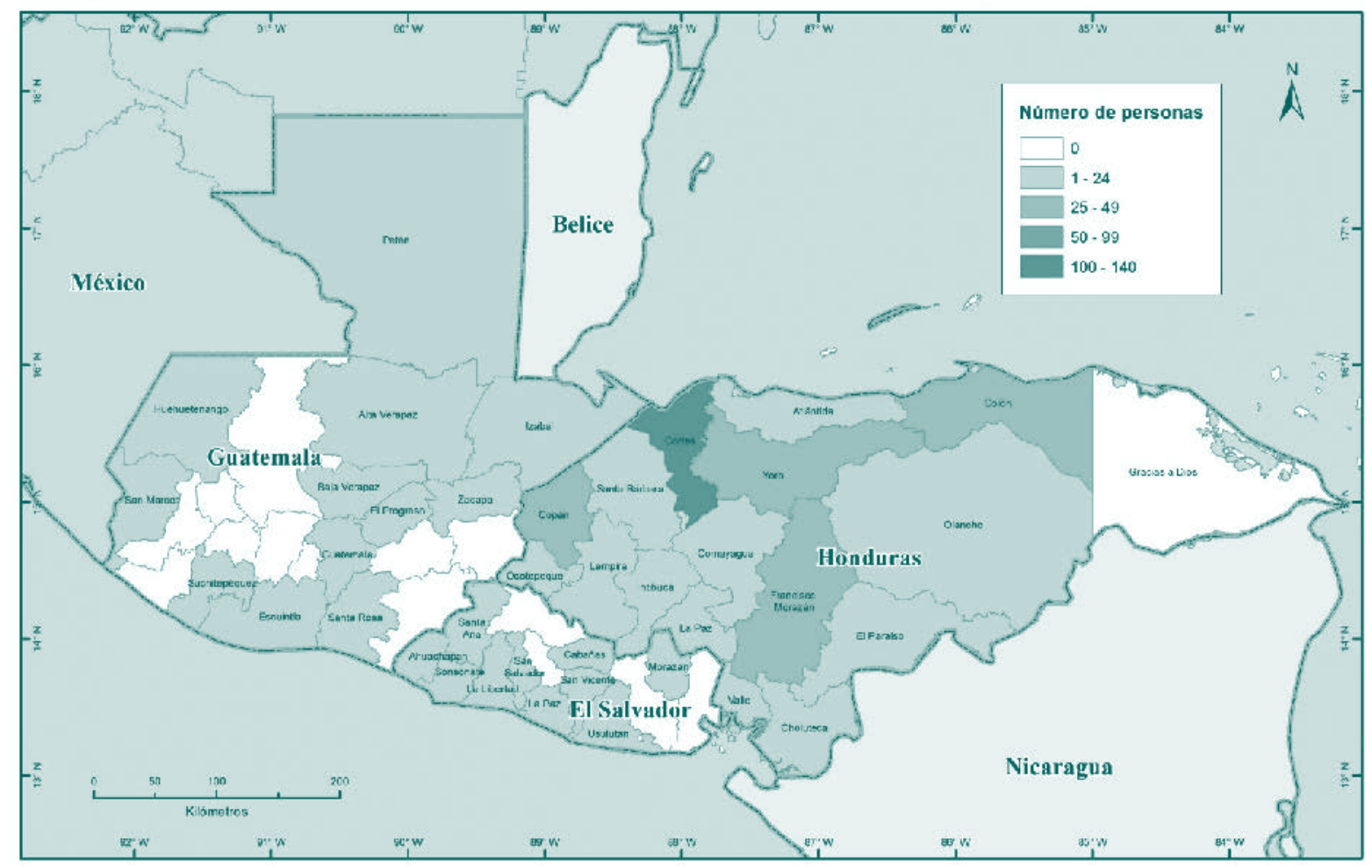

Fuente: elaborado por Gustavo A. Vázquez de El Colef, Monterrey con los resultados del cuestionario aplicado a migrantes en Piedras Negras, Coahuila, en febrero de 2019.

El Mapa №1 muestra la distribución del número de migrantes según el departamento y país de residencia al momento de unirse a la caravana. El mayor número de migrantes que se unieron a la caravana en Honduras, residían en el departamento de Cortés, seguido en nivel de importancia los departamentos de Yoro, Colón, Francisco Morazán y Copán. En El Salvador los departamentos con mayor número de emigrantes fueron San Salvador y Sonsonate y en Guatemala destacaron San Marcos y Guatemala.

En términos de escolaridad predominó el nivel básico con la mitad de las observaciones (49.7\%), seguida del nivel escolar secundario con un cuarto de las observaciones (25.5\%). No se presentaron diferencias significativas en la escolaridad al estratificar por sexo a las personas (Cuadro $\mathrm{N}^{\circ} 1$ ).
Respecto al estado civil la mitad de los migrantes manifestaron ser solteros (49.4\%) predominando en esta categoría los hombres respecto de las mujeres ( $51.7 \%$ vs $43.3 \%)$. También hubo una mayor proporción de mujeres (11.3\%) que de hombres $(4.1 \%)$ en la categoría de separadas o divorciadas.

En términos de la ocupación, los hombres se dedicaban a actividades elementales ${ }^{6}$ en $29.5 \%$, agrícolas (23\%) y artesanales (17.5\%), que en conjunto representan el $70 \%$ de las observaciones. En mujeres el grueso de la población se concentró en actividades de servicios personales (54.1\%), seguido por el rubro de comerciantes (25.6\%) y operadoras de maquinaria (10.5\%), concentrando en conjunto $90.2 \%$ de las observaciones.

6 Las actividades elementales clasifican a los trabajadores que auxilian los procesos productivos realizando actividades sencillas y rutinarias que implican básicamente esfuerzo físico, destreza motriz y conocimientos básicos que se aprenden en la práctica en solo unas jornadas de trabajo. Incluye a los trabajadores de apoyo a: actividades agropecuarias, forestales, pesca y caza, en la industria, ayudantes de conductores de transporte, conductores de transporte de tracción humana y animal y cargadores, en la preparación de alimentos, vendedores ambulantes, trabajadores domésticos, planchadores y otros trabajadores de limpieza, trabajadores de paquetería, de apoyo para espectáculos y repartidores de mercancía (INEGI, 2011, pp. 255-274). 


\section{Cuadro $\mathrm{N}^{\circ} 1$ Características sociodemográficas de los integrantes de la caravana de migrantes centroamericanos en febrero de 2019}

\begin{tabular}{|l|c|c|c|}
\hline \multicolumn{1}{|c|}{ Características } & $\begin{array}{c}\text { Mujeres } \\
\mathbf{n}(\%)\end{array}$ & $\begin{array}{c}\text { Hombres } \\
\mathbf{n}(\%)\end{array}$ & $\begin{array}{c}\text { Total } \\
\mathbf{n}(\%)\end{array}$ \\
\hline País de nacimiento & $120(81.1)$ & $299(77.5)$ & $419(78.5)$ \\
\hline Honduras & $20(13.5)$ & $48(12.4)$ & $68(12.7)$ \\
\hline El Salvador & $5(3.4)$ & $27(7.0)$ & $32(6.0)$ \\
\hline Guatemala & $3(2.0)$ & $12(3.1)$ & $15(2.8) \neq$ \\
\hline Otro & & & \\
\hline Escolaridad & $10(8.5)$ & $17(5.4)$ & $27(6.2)$ \\
\hline Ninguna & $53(44.9)$ & $163(51.4)$ & $216(49.7)$ \\
\hline Primaria & $31(26.3)$ & $80(25.2)$ & $111(25.5)$ \\
\hline Secundaria & $20(16.9)$ & $44(13.9)$ & $64(14.7)$ \\
\hline Bachillerato & $4(3.4)$ & $13(4.1)$ & $17(3.9) \neq$ \\
\hline Profesional & $68(45.3)$ & $172(44.2)$ & $240(44.4)$ \\
\hline Estado civil & $17(11.3)$ & $16(4.1)$ & $33(6.1)$ \\
\hline Vive en pareja & $65(43.3)$ & $201(51.7)$ & $267(49.4) \dagger$ \\
\hline Separado, divorciado & & & \\
\hline Soltero & $2(1.5)$ & $2(0.5)$ & $4(0.8)$ \\
\hline Ocupación & $1(0.8)$ & $6(1.6)$ & $7(1.4)$ \\
\hline Profesionistas & $34(25.6)$ & $42(11.5)$ & $76(15.2)$ \\
\hline Administrativos & $72(54.1)$ & $27(7.4)$ & $99(19.8)$ \\
\hline Comerciantes & $2(1.5)$ & $84(23.0)$ & $86(17.2)$ \\
\hline Servicios personales & $0(0.0)$ & $65(17.5)$ & $65(13.0)$ \\
\hline Actividades agrícolas & $14(10.5)$ & $32(8.7)$ & $46(9.2)$ \\
\hline Actividades artesanales & $8(6.0)$ & $108(29.5)$ & $116(23.2) *$ \\
\hline Operadores maquinaria & & & \\
\hline Actividades elementales & & & \\
\hline
\end{tabular}

Fuente: elaborada con resultados del cuestionario aplicado a migrantes en Piedras Negras, Coahuila, en febrero de 2019.

Contestaron la pregunta 435 personas de un total de $540 . \neq N S ; \& \leq 0.05 ; \dagger p<0.05 ;{ }^{*} p<0.001$

El Cuadro N² presenta las características demográficas de los migrantes en su movilidad. Las mujeres tienden, en mayor proporción que los hombres, a viajar acompañadas, viajar con dos o más personas, viajar con un mayor número de menores, viajar con un mayor número de familiares, viajar con su pareja y viajar con hijos.
La principal razón para migrar fue la falta de empleo en $64.6 \%$ de los casos, seguida de violencia $(16.1 \%)$ e inseguridad (14.1\%), sin mostrar diferencias por sexo. Las mujeres viajaban por primera vez en mayor proporción que los hombres $(94.0 \%$ vs $84.1 ; \mathrm{p}<0.05)$. Un poco más de la mitad de los migrantes dijeron tener familiares en EU (52.6\%), sin mostrar diferencias por sexo. 
Cuadro N² Características sociodemográficas de acompañamiento en la movilidad de los integrantes de la caravana de migrantes centoamericanos en febrero de 2019

\begin{tabular}{|c|c|c|c|}
\hline Características & $\begin{array}{c}\text { Mujeres } \\
\text { n (\%) }\end{array}$ & $\begin{array}{c}\text { Hombres } \\
\text { n (\%) }\end{array}$ & $\begin{array}{l}\text { Total } \\
\text { n (\%) }\end{array}$ \\
\hline \multicolumn{4}{|l|}{ Forma en que viaja } \\
\hline Solo & $32(21.3)$ & $258(73.3)$ & $317(58.8)$ \\
\hline Acompañado & $118(78.7)$ & $104(26.7)$ & $222(41.2)^{*}$ \\
\hline \multicolumn{4}{|c|}{ Personas con que viaja $\neq$} \\
\hline 1 & $44(38.3)$ & $53(52.0)$ & $97(44.7)$ \\
\hline 2 & $29(25.2)$ & $14(13.7)$ & $43(19.8)$ \\
\hline 3 y más & $42(36.5)$ & $35(34.3)$ & $77(35.5) \dagger$ \\
\hline \multicolumn{4}{|c|}{ Menores con que viaja } \\
\hline 0 & $19(17.9)$ & $39(41.5)$ & $58(29.0)$ \\
\hline 1 & $49(46.2)$ & $31(33.0)$ & $80(40.0)$ \\
\hline 2 & $21(19.8)$ & $12(12.8)$ & $33(16.5)$ \\
\hline 3 o más & $17(16.0)$ & $12(12.8)$ & $29(14.5) \dagger$ \\
\hline \multicolumn{4}{|c|}{ Familiares con que viaja } \\
\hline 0 & $8(8.1)$ & $22(23.9)$ & $30(15.7)$ \\
\hline 1 & $40(40.4)$ & $41(44.6)$ & $81(42.4)$ \\
\hline 2 & $19(19.2)$ & $11(12.0)$ & $30(15.7)$ \\
\hline 3 o más & $32(32.3)$ & $18(19.6)$ & $50(26.2) \dagger$ \\
\hline \multicolumn{4}{|l|}{ Viaja con pareja } \\
\hline Sí & $46(59.7)$ & $28(42.4)$ & $74(51.7)$ \\
\hline No & $31(40.3)$ & $38(57.6)$ & $69(48.3) \dagger$ \\
\hline \multicolumn{4}{|l|}{ Viaja con hijos } \\
\hline Sí & $95(89.6)$ & $36(53.7)$ & $131(75.7)$ \\
\hline No & $11(10.4)$ & $31(46.3)$ & $42(24.3)$ * \\
\hline
\end{tabular}

Fuente: elaborada con resultados del cuestionario aplicado a migrantes en Piedras Negras, Coahuila, en febrero de 2019.

Solo contempla a las personas que viajaban acompañadas.

$$
\dagger p<0.05 ;{ }^{*} p<0.001
$$


Cuadro $N^{\circ} 3$ Razones para migrar y perspectivas migratorias de los integrantes de la caravana de migrantes centroamericanos en febrero de 2019

\begin{tabular}{|l|c|c|c|}
\hline \multicolumn{1}{|c|}{ Características } & $\begin{array}{c}\text { Mujeres } \\
\mathbf{n}(\%)\end{array}$ & $\begin{array}{c}\text { Hombres } \\
\mathbf{n}(\%)\end{array}$ & $\begin{array}{c}\text { Total } \\
\mathbf{n}(\%)\end{array}$ \\
\hline $\begin{array}{l}\text { Causa principal por la que salió de su } \\
\text { país: }\end{array}$ & & & \\
\hline Falta de empleo & $96(64.0)$ & $252(64.8)$ & $348(64.6)$ \\
\hline Inseguridad & $16(10.7)$ & $60(15.4)$ & $76(14.1)$ \\
\hline Violencia & $26(17.3)$ & $61(15.7)$ & $87(16.1)$ \\
\hline Otro & $12(8.0)$ & $16(4.1)$ & $28(5.2) \neq$ \\
\hline ¿Primera vez que viaja a EU? & & & \\
\hline Sí & $141(94.0)$ & $327(84.1)$ & $468(86.8)$ \\
\hline No & $9(6.0)$ & $62(15.9)$ & $71(13.2) \dagger$ \\
\hline Familiares en EU & & & \\
\hline Sí & $85(57.0)$ & $197(50.9)$ & $282(52.6)$ \\
\hline No & $64(43.0)$ & $190(49.1)$ & $254(47.4) \neq$ \\
\hline Estando en Piedras Negras (PN) & & & \\
¿qué piensa hacer? & & & $193(3)$ \\
\hline Solicitar asilo en EU & $74(54.8)$ & $123(33.3)$ & $197(39.1)$ \\
\hline Cruzar a EU & $27(20.0)$ & $77(20.9)$ & $104(20.6)$ \\
\hline Quedarse en PN & $23(17.0)$ & $86(23.3)$ & $109(21.6)$ \\
\hline Ir a otro lugar en México & $7(5.2)$ & $69(18.7)$ & $76(15.1)$ \\
\hline Regresar a su origen & $0(0.0)$ & $5(1.4)$ & $5(1.0)$ \\
\hline Otro & $4(3.0)$ & $9(2.4)$ & $13(2.6)$ \\
\hline Regresar a su origen & $3(2.0)$ & $17(4.4)$ & $20(3.7)$ \\
\hline Sí & $144(96.0)$ & $366(94.3)$ & $510(94.8)$ \\
\hline No & $3(2.0)$ & $5(1.3)$ & $8(1.5) \neq$ \\
\hline No sabe & $127(84.7)$ & $305(79.2)$ & $432(80.7)$ \\
\hline Trámite migratorio en México: & $23(15.3)$ & $80(20.8)$ & $103(19.3) \neq$ \\
\hline Sí & $122(96.8)$ & $293(96.1)$ & $415(96.3)$ \\
\hline No & $3(2.4)$ & $11(3.6)$ & $14(3.2)$ \\
\hline Proceso iniciado & $1(0.8)$ & $0(0.0)$ & $1(0.2)$ \\
\hline Regularización humanitaria & & $1(0.3)$ & $1(0.2) \neq$ \\
\hline Solicitud de refugio & & & \\
\hline Regularización familiar & & & \\
Regularización por empleo & & & \\
\hline
\end{tabular}

Fuente: elaborada con resultados del cuestionario aplicado a migrantes en Piedras Negras, Coahuila, en febrero de 2019.

solo contempla a las personas que viajaban acompañadas.

$$
\neq N S ; \& \leq 0.05 ; \uparrow p<0.05 ;{ }^{*} p<0.001
$$


Mapa N² Departamentos en Centroamérica y Estados en México en que los migrantes se unieron a la caravana

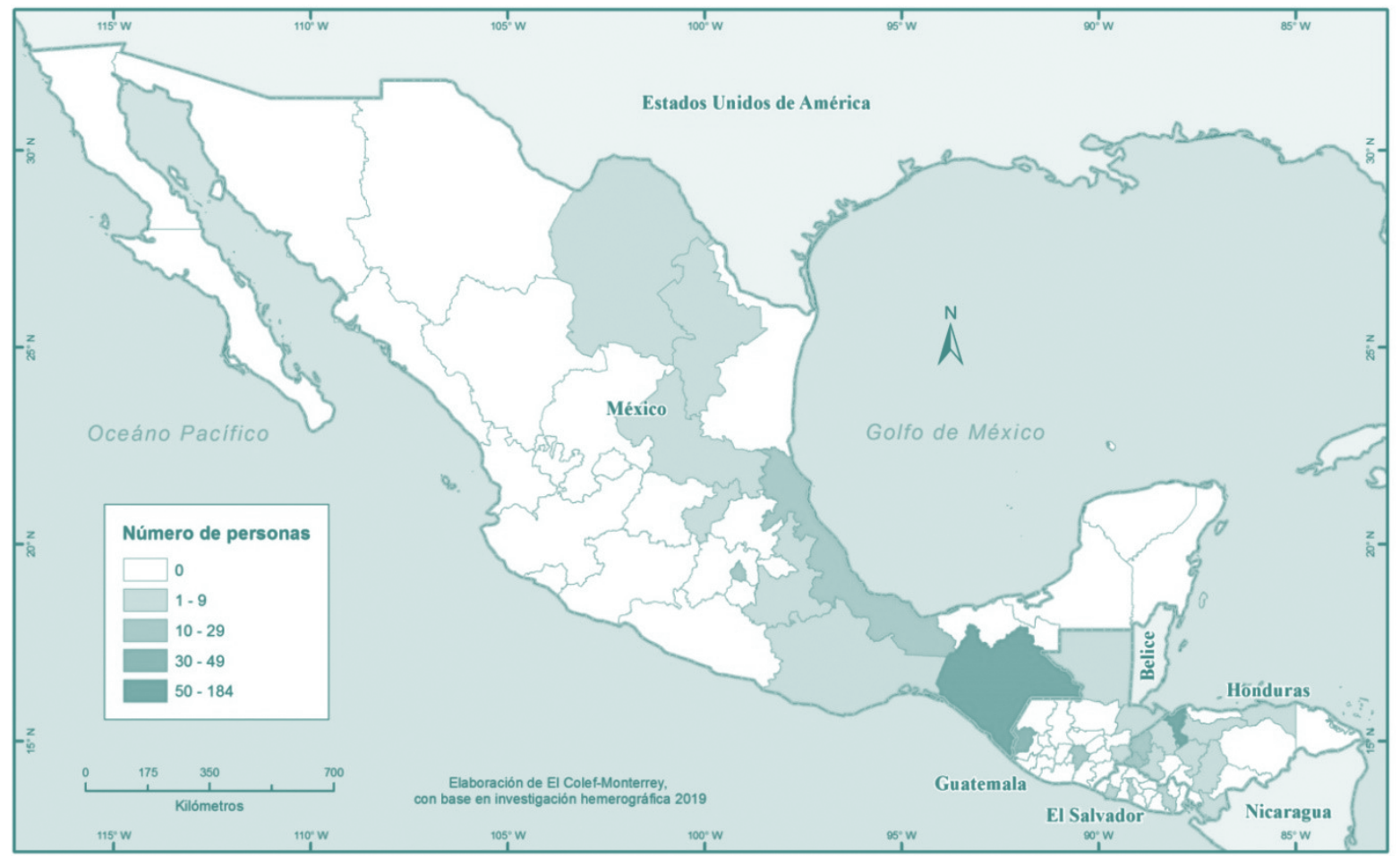

Fuente: elaborado por Gustavo A. Vázquez de El Colef, Monterrey con los resultados del cuestionario aplicado a migrantes en Piedras Negras, Coahuila, en febrero de 2019.

\subsection{Dinámica migratoria}

Respecto a las decisiones que deben tomar, la mayor proporción considera la posibilidad de solicitar asilo en EU (39.1\%), cruzar a EU (20.6\%), quedarse en Piedras Negras $(21.6 \%)$ e irse a vivir a otra parte de México (15.1\%). Las mujeres tuvieron una proporción mayor que los hombres en la opción de solicitar asilo en EU mientras que los hombres en mayor proporción que las mujeres mostraron el deseo de quedarse en Piedras Negras o ir a otros lugares de México $(p<0.05)$.

Apenas el $2 \%$ de las mujeres y el $4.4 \%$ de los hombres mostraron el deseo de regresar a su país de origen, mientras que una proporción relativamente mayor de mujeres habían iniciado algún trámite migratorio en México respecto de los hombres (84.7\% vs $79.2 \% ; p>0.05)$. El trámite más frecuente fue el de la regularización por razones humanitarias en el $96.3 \%$ de los casos, muy pocos migrantes solicitaron las figuras de refugio (3.2\%), reunificación familiar $(0.2 \%)$ y regularización por trabajo $(0.2 \%)$.

El Mapa `2 muestra la distribución del número de migrantes desagregado por departamento en Centroamérica o estado en México al momento de unirse a la caravana. Pueden observarse tres puntos importantes de unión con la caravana. En el caso de Honduras el departamento de Cortés en Honduras, Tecún Humán en Guatemala y el estado de Chiapas en México.

\section{Conclusiones}

Nuevos patrones de migración desde Centroamérica hacia Estados Unidos a través de México se hicieron notar en los medios de comunicación desde febrero de 2018. Se trata de movimientos migratorios que hacen visible su camino al organizar su desplazamiento en forma de caravana. Aunque Martínez Hernández-Mejía (2018) refiere que las formas actuales más conocidas de desplazamiento de migrantes son las vías crucis y las caravanas, en este trabajo haremos alusión al segundo término.

La conformación de la caravana de migrantes centroamericanos se vio favorecida, además de las condiciones contextuales de los países de origen, por la difusión desde 2013 de información en Centroamérica sobre la posibilidad de llegar a EU a través de una petición de asilo (Solalinde y Correa, 2019), la propuesta del gobierno mexicano en octubre de 2018 sobre un programa de visas temporales de trabajo a migrantes centroamericanos (El Colef, 2019) 
y la difusión hacia finales de diciembre de 2018 de la salida de una caravana en la zona metropolitana de San Pedro Sula en Honduras en enero de 2019 (El Heraldo, 2018).

Consistente con lo anterior es el reporte de la Organización Internacional para las Migraciones (OIM) sobre la situación de flujo de personas migrantes en tránsito entre el 15 y el 22 de enero de 2019, en que se informaba de un flujo de 5,087 personas migrantes evaluadas en su paso por Tecún Umán, Guatemala (OIM, 2019a). De este flujo de personas que ingresaron a México en enero de este año aproximadamente 1,800 arribaron a la ciudad de Piedras Negras, Coahuila, el 4 de febrero.

Los resultados de este estudio muestran la dinámica demográfica en la conformación de la caravana. Si su punto de partida estuvo situado en la zona urbana de San Pedro Sula, departamento de Cortés, los departamentos de Yoro, Colón, Francisco Morazán y Copán fueron también punto de partida de migrantes en Honduras. En El Salvador destacaron los departamentos de San Salvador y Sonsonate y en Guatemala fueron San Marcos y Guatemala. Asimismo, tres fueron los puntos de mayor relevancia en que los migrantes se unieron a la caravana, el departamento de Cortés en Honduras donde el punto de partida de la caravana si situó en San Pedro Sula, el departamento de San Marcos en Guatemala donde destaca la ciudad de Tecún Humán en la frontera con México y el estado de Chiapas en México donde destacan Tapachula y Ciudad Hidalgo.

La caracterización socio-demográfica de los integrantes de la caravana de migrantes centroamericanos en Piedras Negras, Coahuila, es consistente con el reporte de la caravana que arribó a la ciudad de Tijuana en octubre de 2018 (El Colef) y también con el perfil de los migrantes que fue analizado por la OIM en enero de 2019 en una muestra de 819 migrantes estudiados en Suchiate, Chiapas (OIM, 2019a). Se trata de una población joven, con 2.5 hombres por cada mujer, provenientes principalmente de Honduras, con una escolaridad dominante en el nivel básico y secundario. Entre los hombres predominaron las actividades elementales y de apoyo, agrícolas y artesanales, mientras que en mujeres predominaron actividades de servicios personales, comerciantes y operadoras de maquinaria.

Fue notoria una diferencia de sexo en el desplazamiento de la población y un ingreso tardío de las mujeres en los procesos migratorios. Los resultados muestran que las mujeres viajan por primera vez en mayor proporción que los hombres y que el acompañamiento en el proceso migratorio representa una estrategia de defensa ante la vulnerabilidad que tradicionalmente han enfrentado las mujeres migrantes centroamericanas (Willers, 2016; Cueva-Luna y Terrón-Caro, 2014; Semple y Malkin, 2018).

Los resultados de este trabajo denotan los graves problemas económicos y sociales por los que atraviesan los países centroamericanos. La principal causa que se adujo para salir de su país fue la falta de empleo, seguida de la presencia de violencia e inseguridad. Estos resultados coinciden con los encontrados por la OIM, en migrantes centroamericanos en el Suchiate, Chiapas (OIM, 2019b), donde el mejoramiento de las condiciones socio-económicas, protección y seguridad, fueron las necesidades inmediatas identificadas para continuar con su proyecto migratorio.

Finalmente se considera que el incremento de la población centroamericana en EU ha fomentado la conformación de redes de atracción a la inmigración (Gonzáles et al., 2017) pues más de la mitad de las personas entrevistadas en este estudio dijeron tener familiares en ese país y dicho fenómeno puede estar relacionado con un incremento en la búsqueda de asilo visualizado principalmente por las mujeres. Asimismo, la mayor parte de las personas entrevistadas buscan llegar de una u otra forma a EU con pocos casos que buscan residir en México o regresar a su país de origen.

\section{Referencias bibliográficas}

- Castillo, M. A. y Toussaint, M. (2015). La frontera sur de México: orígenes y desarrollo de la migración centroamericana. Cuadernos Intercambio sobre Centroamérica y el Caribe, 12(2), 59-86.

- Cueva-Luna, T. E., \& Terrón-Caro, T. (2014). Vulnerabilidad de las mujeres migrantes en el cruce clandestino por Tamaulipas-Texas. Papeles de población, 20(79), 209-241.

- El Colegio de la Frontera Norte. (2018). La caravana de migrantes centroamericanos en Tijuana 2018. Diagnóstico y propuestas de acción. Tijuana: El Colegio de la Frontera Norte.

- El Colegio de la Frontera Norte. (2019). La caravana centroamericana de migrantes en Piedras Negras, Coahuila 2019. Diagnóstico y propuestas de acción. Tijuana: El Colegio de la Frontera Norte. 
- El Heraldo. (28 de diciembre de 2018). Organizan una nueva caravana migrante desde Honduras hacia Estados Unidos. El Heraldo. Recuperado de https://www.elheraldo.hn/pais/1245594-466/organizan-una-nueva-caravana-migrante-desde-honduras-hacia-estados-unidos

- González, J. E., Zapata, R., Anguiano, M. E. (2017). Migración centroamericana en tránsito por México. En Consejo Nacional de Población (Autor), La situación demográfica de México 2016 (p. 221-232). México: CONAPO.

- INEGI, I. (2011). Sistema Nacional de Clasificación de Ocupaciones 2011: SINCO. México: INEGI.

- Lesser, G. y Batalova, J. (2017). Inmigrantes Centroamericanos en los Estados Unidos. Migration Policy Institute. Recuperado de https://www.migrationpolicy.org/article/inmigrantes-centroamericanos-en-los-estados-unidos

- López Recinos, V. (2013). Desarrollo, migración y seguridad: El caso de la migración hondureña hacia Estados Unidos. Migración y Desarrollo, 11(21), 65-105.

- Lwanga SK, Lemeshow S. \& World Health Organization. (1991). Sample size determination in Health: A practical manual. Geneva: WHO.

- Martínez Hernández-Mejía, I. (2018). Reflexiones sobre la caravana migrante. Recuperado de https://rei.iteso.mx/bitstream/handle/11117/5616/ S3\%20Reflexiones\%20sobre\%20la\%20caravana\%20migranteAjustado.pdf?sequence $=2$

- Martínez, G., Cobo, S. D., \& Narváez, J. C. (2015). Trazando rutas de la migración de tránsito irregular o no documentada por México. Perfiles Latinoamericanos, 23(45), 127-155.

- Menjívar, C. y Gómez Cervantes, A. (2018). El Salvador: Civil war, natural disasters, and gang violence drive migration Washington, DC: Migration Policy Institute.

- Organización Internacional para las Migraciones (OIM). (2019a). Reporte Situacional de Flujos de Personas Migrantes en Tránsito (15-22 enero). Recuperado de https://migration.iom.int/system/ tdf/reports/Caravana\%20-\%20Ronda\%204\%20 Reporte\%20Situacional\%209.pdf?file=1\&type=node\&id $=5026$
- OIM. (2019b). México: Monitoreo de flujos migratorios en Suchiate, Chiapas (25 al 30 de enero). Recuperado de https://www.refworld.org.es/docid/5cdc59774.html

- París, P. M. D., Ley, C. M. y Peña, M. J. (Autores). (2016). Migrantes en México Vulnerabilidad y Riesgos. Ginebra: Organización Internacional para las Migraciones (OIM), El Colegio de la Frontera Norte.

- Pierce, S. (2015). Unaccompanied child migrants in US communities, immigration court, and schools. Washington, DC: Migration Policy Institute.

- Ramos, T. E., Campos Moran, S., Jasmin Bolaños, H., \& Calles Minero, C. (2013). Perfil Actual de la Persona Migrante en El Salvador. Washington, D. C.: USCRI/ Universidad Tecnológica de El Salvador.

- Schiavon, J. (2015). La migración Centroamérica-México-Estados Unidos: historias de vulnerabilidad y políticas inacabadas. Con-Temporánea, 2(4).

- Semple, K. y Malkin, E. (1 de noviembre de 2018). La caravana migrante inspira a más centroamericanos que buscan viajar a Estados Unidos. The New York Times. Recuperado de https://www. nytimes.com/es/2018/11/01/caravanas-migrantes-centroamerica-mexico/

- Solalinde, A. y Correa-Cabrera, G. (5 de mayo de 2019). Caravanizando la Migración: Una perspectiva desde México. Proceso. Recuperado de https://www.proceso.com.mx/582414/caravanizando-la-migracion-una-perspectiva-desde-mexico

- Willers, S. (2016). Migración y violencia: las experiencias de mujeres migrantes centroamericanas en tránsito por México. Sociológica (México), 31(89), 163-195.

- Zuñiga Nuñez, M. (2016). Migración, Pandillas y Criminalización: La conflictividad social estadounidense y su relación con El Salvador. En C. Sandoval (edit). Migraciones en América Central Políticas, territorios y actores (p. 25-44). San José, Costa Rica: UCR. Recuperado de http://www. editorial.ucr.ac.cr/ciencias-sociales/item/2162-migraciones-en-america-central-politicas-territorios-y-actores.html 


\section{Anexos}

\section{Anexo $\mathrm{N}^{\circ} 1$}

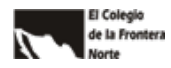

Le solicitamos su apoyo, respondiendo a las preguntas de esta encuesta realizada por El Colegio de la Frontera Norte (centro educativo mexicano). La participación en esta encuesta es absolutamente confidencial y voluntaria. La información que usted nos proporcione se manejará de manera anónima y sin utilizar su identidad personal. Tanto si decide responder a la encuesta como si no, esto no le perjudicará ni beneficiará de ningún modo y su decisión será plenamente respetada.

Condiciones de movilidad y estancia de la población integrante de la caravana migrante $\quad$ FOLIO

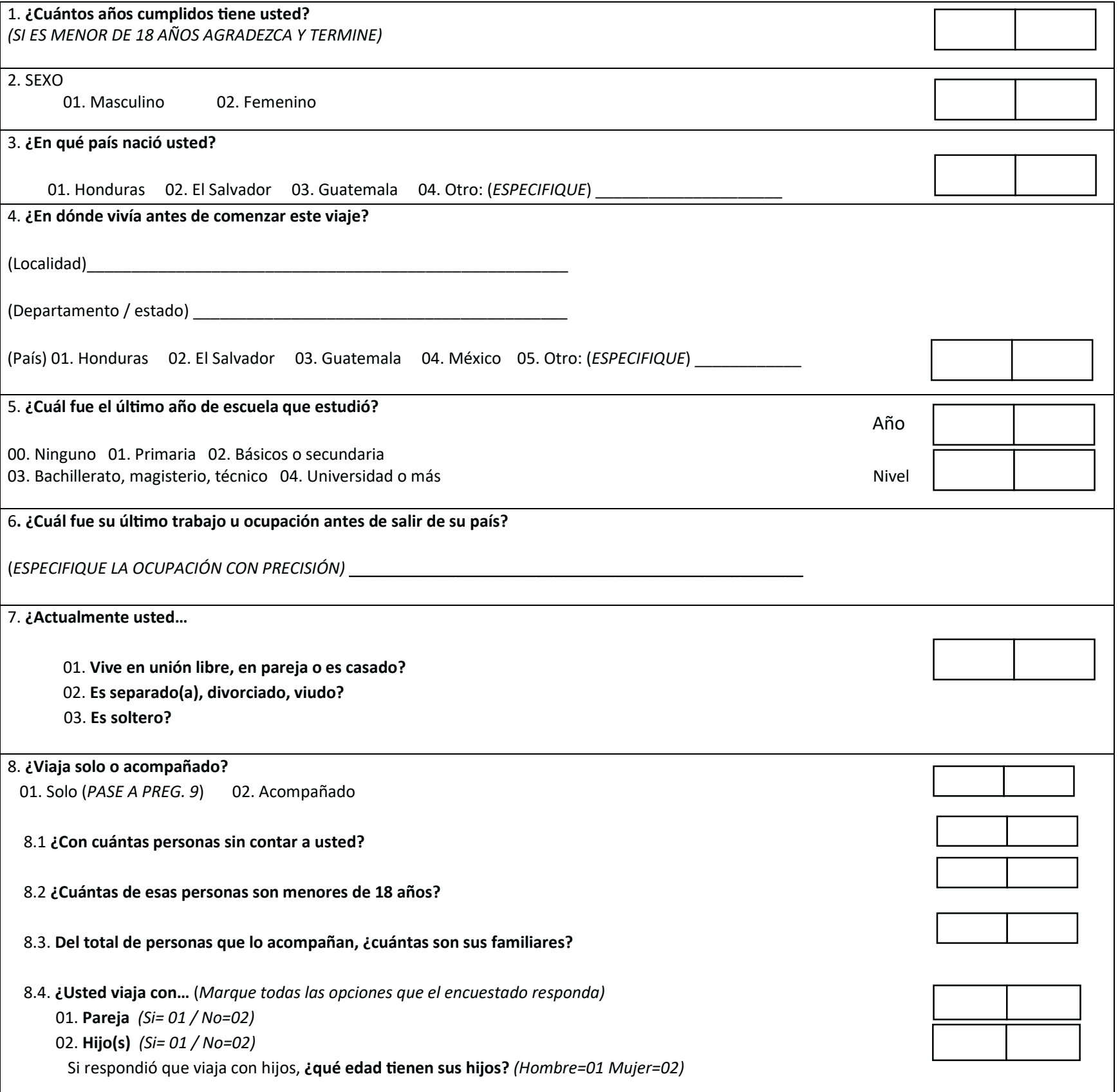


Para cada hijo/a anotar sexo y edad

si son más de 4 hijos anotar sexo y edad aquí
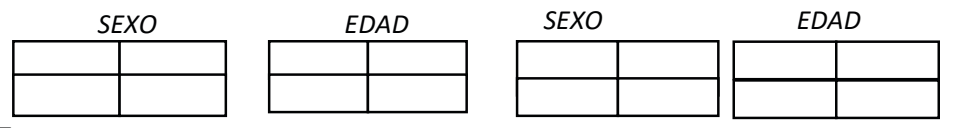

03. Hermanos ( $\mathrm{Si}=01 / \mathrm{No}=02)$

04. Otros familiares (primos, tíos, abuelos) ( $\mathrm{Si}=01 / \mathrm{No}=02$ )

05. Amigos ( $\mathrm{Si}=01 / \mathrm{No}=02)$

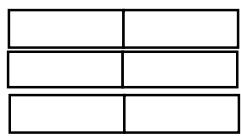

\section{9. ¿Usted llegó a Piedras Negras con la caravana?}

01. Sí 02. No (PASE A PREG.10)

\section{1 ¿En qué lugar se sumó a la caravana por primera vez?}

(Localidad)

(Departamento / estado)

(País) 01. Honduras

02. El Salvador

03. Guatemala

04. México

05. Otro: (ESPECIFIQUE)

9.2 ¿Cuándo se sumó a la caravana? Día:

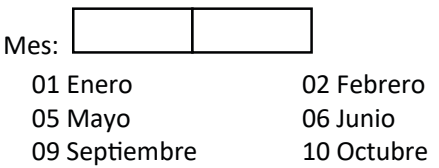

03 Marzo
07 Julio
11 Noviembre

04 Abril

08 Agosto

09 Septiembre $\quad 10$ Octubre 11 Noviembre 12 Diciembre

10. ¿Me puede decir las 2 razones más importantes del por qué salió de su último lugar de residencia? (ESCUCHE Y CODIFIQUE)

01. Falta de empleo o medios económicos

02. Inseguridad (robos, delincuenca, etc)

03. Violencia causada por pandillas o maras

04. Violencia doméstica

05. Violencia de género o discriminación por preferencia sexual

06. Evitar extorsiones

07. Presiones políticas (persecusión política)

08. Persecución o discriminación religiosa

09. Desastre natural (terremoto, inundaciones, erupción de volcán, etc) o sequía

10. Proyectos de desarrollo (hidroeléctricas, minas, desarrollo turísticos, aeropuertos, carreteras, etc)

11. Reunirse con su familia

12. Otra (ESPECIFIQUE)

11. ¿Es la primera vez que viaja a Estados Unidos?

01. Sí (PASE A PREG.12) 02. No

11.1 ¿Cuántas veces ha intentado cruzar a Estados Unidos?

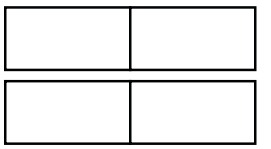

11.2 ¿Cuántas veces ha logrado cruzar a Estados Unidos?

12. ¿Tiene familiares en Estados Unidos?
01. Sí
02. No

13. ¿Durante el trayecto por México, usted fue víctima de alguna agresión o maltrato por parte de alguna autoridad mexicana?
01. Sí
02. No (PASE A PREG. 14)

13.1. ¿Por parte de qué autoridad? (ANOTE HASTA 2 AUTORIDADES)

01. Instituto Nacional de Migración (INM)

02. Grupo Beta

03. Policía Federal

04. Policía Estatal 


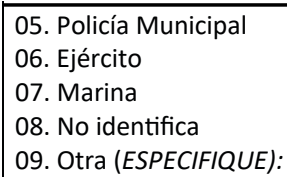

14. ¿Por qué decidieron llegar a Piedras Negras y no a otro lugar de la frontera?

01. El viaje es más corto

02. El líder así lo decidió

03. Porque aquí venía la caravana

04. Porque esta frontera es más segura

05. Porque le proporcionaron transporte

06 Porque es más fácil el cruce

07. Otra (ESPECIFIQUE)

\section{1. Ahora que está en Piedras Negras, ¿qué piensa hacer?:}

01. Solicitar asilo en EUA

(Si respondió que piensa solicitar asilo en EUA), 14.1 ¿Ya se anotó en la lista? ( $\mathrm{Si}=01$ / No=02)

02. Cruzar a EUA

03. Quedarse en Piedras Negras

04. Ir a otro lugar de México

05. Regresar a su lugar de origen

06. Otro (ESPECIFIQUE)

15. ¿Le gustaría regresar a su lugar de origen en este momento?

01. Si (PASE A LA PREG.16) 02. No 03. No sabe (PASE A LA PREG.16)

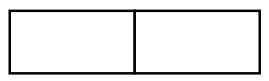

\section{1 ¿Por qué no le gustaría regresar?}

01. Aquí hay más oportunidades

02. Tengo familiares (en México o en EUA) y quiero estar cerca

03. Quiero llegar a EUA

04. No hay trabajo de dónde vengo

05. Por violencia en lugar de origen

06. Tengo miedo de regresar

07. Otro (ESPECIFIQUE)

\section{6. ¿Ha iniciado algún trámite migratorio en México?}

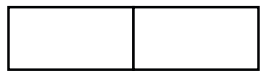

\section{1 ¿Qué proceso ha iniciado?}

01. Regularización por razones humanitarias

02. Solicitud de refugio ante la Comisión Mexicana de Ayuda a

Refugiados (COMAR)

03. Regularización por vínculo familiar

04. Regularización por oferta de empleo

\section{7. ¿Ha solicitado trabajo en Piedras Negras?}

01. Sí

02. No
16.2 ¿Piensa iniciar algún trámite migratorio en México?

01. Sí 02. No (PASE A LA PREG. 17)

03 No sabe (PASE A LA PREG. 17)

16.3 ¿Cuál?:

01. Regularización por razones humanitarias

02. Solicitud de refugio ante la Comisión

Mexicana de Ayuda a Refugiados (COMAR)

03. Regularización por vínculo familiar

04. Regularización por oferta de empleo
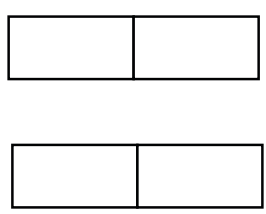

18. ¿Actualmente trabaja?

01. Sí 02. No (PASE A LA PREG. 19)

18.1. ¿En qué trabaja? 


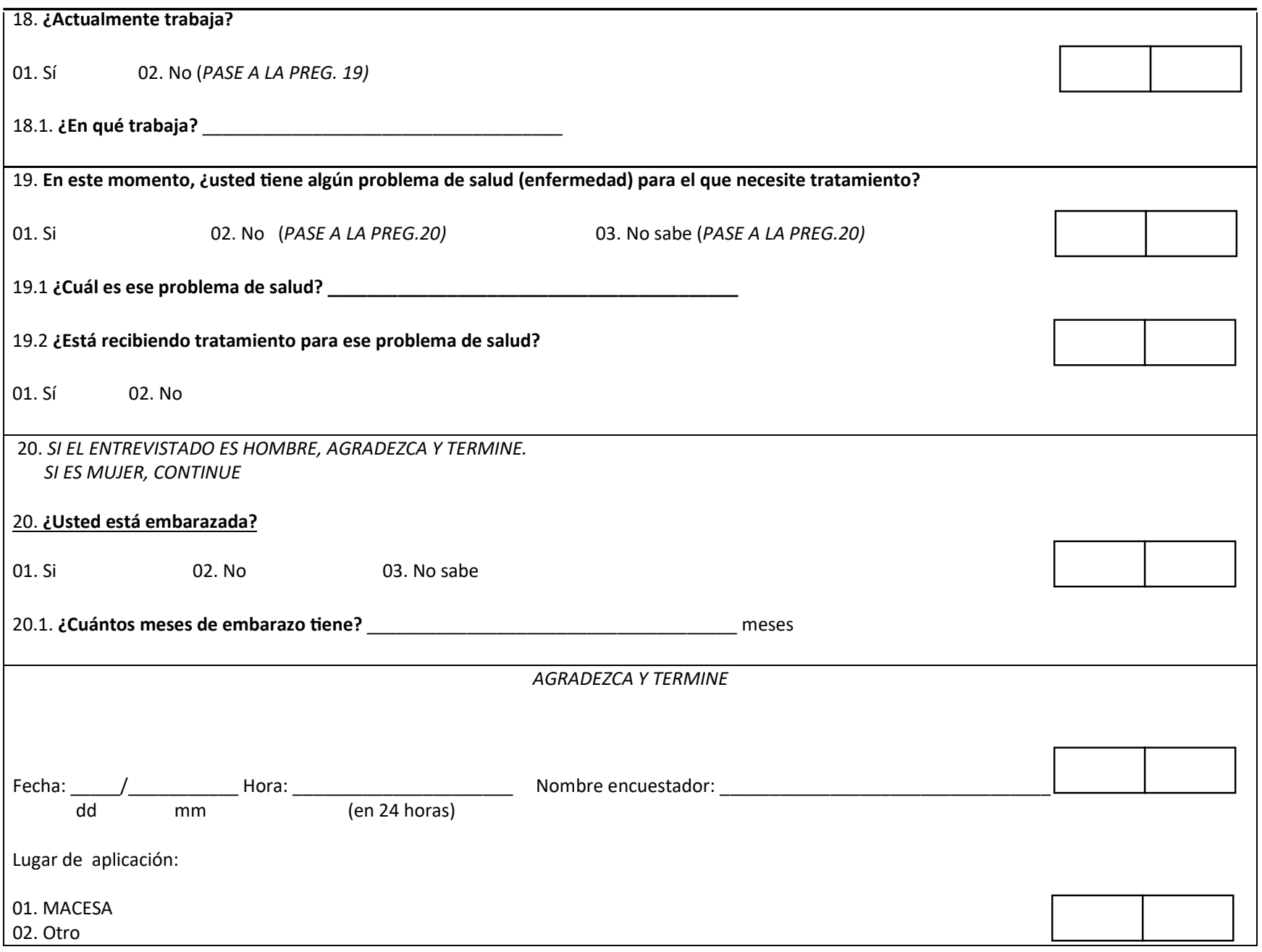

